\title{
Populismusforschung: Blick über die Grenzen mit vielen Anregungen
}

Lucardie, Paul und Gerrit Voerman: Populisten in de polder, Boom Onderwijs, Amsterdam 2012, 168 Seiten, $€ 18,50$.

Reynié, Dominique: Populismes: la pente fatale, Editions Plon, Paris 2011, 280 Seiten, € 20,-.

Taguieff, Pierre-André: Le noveau national-populisme, CNRS Editions, Paris 2012, 128 Seiten, $€ 6,10$.

Fachzeitschrift „Cités. Philosophie, Politique, Histoire”: Le Populisme, contre les peuples?, 49. Jg. (2012), H. 1.

Fachzeitschrift „Critique”: Populismes, 67. Jg. (2012), H. 776/777.

Fachzeitschrift „Politique. Revue de débats”: Les nouveaux habits du populisme, 16. Jg. (2012), H. 75 .

Konzepte sind nicht nur in der Politik oder in der Wirtschaft wichtig, sondern auch von herausragender Bedeutung innerhalb der Sozialwissenschaften. Sie helfen, logische Strukturen zu schaffen, um am Ende eine fundierte Bewertung des Untersuchungsgegenstandes vornehmen zu können. Wie gut und tragfähig ein Konzept wirklich ist, hängt nicht nur von unstrittig wichtigen Merkmalen wie empirische und theoretische Relevanz, Regelsetzung und Klarheit ab. ${ }^{1}$ Kann ein politischer Kampfbegriff wie Populismus überhaupt ein Konzept sein? Er hat den Nachteil, durch seine emotionale Aufgeladenheit eine wissenschaftliche Analyse zu beeinträchtigen. Ein Kampfbegriff par excellence ist der Populismus, bei dem jeder, gleich ob Politiker oder Medienkonsument, irgendwie zu wissen glaubt, was gemeint ist. Das jeweils Gemeinte und Assoziierte bleibt diffus, sogar widersprüchlich, ist offenbar nur schwer auf den Punkt zu bringen. Das liegt auch daran, dass der Populismusbegriff unterschiedliche Facetten besitzt und der Alltagssprache entnommen ist. In der Politik wie in den Medien wird er undifferenziert im Sinne eines catch-all-terms gebraucht. Populismus wird in wissenschaftlichen Konferenzen gemeinhin als Gefahr wahrgenommen, und zwar über politische Lager hinweg. ${ }^{2}$

In der Regel wird Populismus auf Parteien bezogen: Seit den frühen 1980er Jahren können neuartige, in erster Linie rechtspopulistische Parteien mit einer Anti-EstablishmentHaltung, Protestthemen und einer charismatischen Führungspersönlichkeit immer wieder Wahlerfolge auf nationaler Ebene erzielen, so in Frankreich, Österreich, Italien, den Niederlanden, Belgien und Skandinavien, ebenso mit stärkeren Fluktuationen in Ostmitteleuropa,

1 Vgl. Florian Hartleb, Wie entsteht ein gutes sozialwissenschaftliches Konzept?, in: Zeitschrift für Politikberatung, 4. Jg. (2011), H. 3, S. 109 - 118.

2 Vgl. ders., Vier Konferenzen von vier verschiedenen Fraktionen (Grüne, Liberale, Christdemokraten und Sozialisten) zum selben Thema mit dem Titel „Neue Gefahr des Populismu““ im Europäischen Parlament (30./31. März und 21. Juni 2011) in Brüssel, in: ZParl, 42. Jg. (2011), H. 2, S. $466-469$. 
was nahe legt, von einer eigenständigen Parteienfamilie zu sprechen. ${ }^{3}$ Die Eurowährungskrise lässt die von Populisten forcierte „Renationalisierung Europa“ “ als Antwort auf die Pläne einer politischen Union oder von Vereinigten Staaten zumindest möglich erscheinen. ${ }^{4}$ Gerade durch diese empirische Relevanz ist die Populismusforschung auf dem Weg, einen festen Platz in der Politikwissenschaft zu bekommen. ${ }^{5}$ Wie relevant die Forschung wirklich ist, kann ein Blick ins Ausland belegen, wo Sonderhefte zum Populismus en vogue zu sein scheinen. ${ }^{6}$

In Frankreich etwa gibt es eine regelrechte Konjunktur, nachdem Marine Le Pen erfolgreich in die Fußstapfen ihres Vaters tritt und den Front National modernisiert. Seit Jahren werden viele Impulse gesetzt, etwa durch Pierre-André Taguieff. In seinem neuen Büchlein verteidigt er seine Konzeption des Nationalpopulismus, die in Populismus einen Politikstil sieht. Die Diskussion in Frankreich sei Anfang der 1990er entstanden, verbunden nicht nur mit dem Namen Jean-Marie Le Pen, sondern auch mit dem linkspopulistischen Unternehmer Bernard Tapie. Nun gibt es quasi als Nachfolger Le Pens Tochter Marine und auf der linken Seite Jean-Luc Mélenchon. Manchmal könne der Populismus eine Verbindung mit dem Rechtsextremismus, also mit Xenophobie, Intoleranz und Rassismus eingehen, wobei er eher für demagogische Politik stehe. Es gebe auf der gesamten Rechts-Links-Skala von Politik eben viele Varianten des Populismus, weshalb Taguieff viele Erscheinungsformen, etwa auch die Tea-Party in den USA andiskutiert. Die allgemeine Empörungswelle, verbunden mit dem zum Symbol gewordenen Büchlein „Indignez-vous!“ von Stéphane Hessel, lege es im Zeitalter der Unsicherheit auch innerhalb der EU nahe, dass neue Formen von Populismus entstünden. Viele Aspekte sind bereits bekannt, doch gelingt es dem Autor, durch seinen breiten Hintergrund mit seiner Gedankenskizze zum Nachdenken anzuregen.

Dominique Reynié, Professor für Politikwissenschaft bei Sciences Po in Paris, nähert sich dem Phänomen ebenfalls eher assoziativ. In seinem Buch „Populismes: la pente fatale“, das mit dem „Prix du livre politique 2012 et Prix des députés 2012“ ausgezeichnet wurde, sieht er Populismus als Syndrom eines tief sitzenden Identitätskonflikts in Europa, der sich insbesondere in Immigrations- und Integrationsfragen äußert. Populismus ist also auch eine Folge eines Kulturkonflikts, nicht zuletzt mit Blick auf die Frage einer nationalen und europäischen Identität und deren Abgrenzungsrituale. Als Beleg führt er unter anderem die deutsche Sarrazin-Debatte an, die auch international für Aufsehen sorgte. So firmiert der Islam nach dem 11. September 2001 als eine globale Bedrohung, die besonders in den Niederlanden und Österreich im politischen Diskurs artikuliert wird. Die etablierten Parteien werden dadurch unter Druck gesetzt. Reynié bereichert pointiert die Populismusfor-

3 Vgl. Cas Mudde, Populist Radical Right Parties in Europe, Cambridge 2007; Frank Decker I Marcel Lewandowsky, Die rechtspopulistische Parteienfamilie, in: Uwe Jun / Banjamin Höhne (Hrsg.), Parteienfamilien. Identitätsbestimmend oder nur noch Etikett, Opladen / Toronto 2012, S. $268-281$.

4 Vgl. Florian Hartleb, Renationalisierung Europas?, in: Auslandsinformationen der Konrad-Adenauer-Stiftung, April 2012, S. 124 - 139 (in englischer Fassung: Renationalisation of Europe?, in: KAS International Reports, 4/2012, S. 113 - 127).

5 Vgl. die offene Frage des Rezensenten am Ende seiner Dissertation 2004: ders., Rechts- und Linkspopulismus. Eine Fallstudie anhand von Schill-Partei und PDS, Wiesbaden 2004, S. 331.

6 Das gilt mit Einschränkungen auch für Deutschland: Die Fachzeitschrift „Totalitarismus und Demokratie" widmete dem Thema ein Sonderheft, 8. Jg. (2011), H. 2; ebenso die Berliner Debatte Initial, 22. Jg. (2011), H. 1 und die APuZ, 62. Jg. (2012), H. 5/6. 
schung, obwohl ein systematischer Vergleich fehlt, ebenso ein Bezug auf die Extremismusforschung, sei es als Abgrenzung oder Sichtbarmachung von Unterschieden.

Cités widmet sich in einem Schwerpunktheft dem Populismus mit der Frage, ob der angeblich volksnahe Populismus gegen das „Volk“ gerichtet sei. Es ist kein Geheimnis, dass Populisten behaupten, den Volkswillen a priori im politischen Entscheidungsprozess unverfälscht wiederzugeben. Der Tenor liegt auf der Erkenntnis, dass es in Frankreich eine Wiederkehr des Populismus gebe. Dem wird wissenschaftlich auf den Grund gegangen. Guy Hermet, der sich mit einer Monographie, einem Standardwerk zum Populismus in seiner globalen Kraft, einen Namen gemacht hat ${ }^{7}$, behandelt den Populismus in Lateinamerika. Lateinamerika, das historisch als Paradebeispiel und beinahe idealtypische Sonderform des Populismus gilt. In den 1940er und 1950er Jahren kamen nationalistisch-autoritär geprägte populistische Regime auf, die wirtschaftlich und außenpolitisch Unabhängigkeit von den USA und der Kommunistischen Internationalen demonstrieren wollten. Der Regierungsoder Regimepopulismus, mythenhaft und folkloristisch aufbereitet, wuchs zur dominierenden politischen Kraft Lateinamerikas und konnte im Unterschied zu vielen anderen populistischen Erscheinungsformen zu einer dauerhaften Institution mutieren. Aktuell scheint besonders der Fall Hugo Chávez in diesem Kontext eine Rolle zu spielen, wie der Beitrag von Stephan Launay verdeutlicht. Zurück im europäischen Kontext spricht Jean-Pierre Rioux, Herausgeber eines wichtigen Sammelbandes, von der Spannung durch den Populismus ${ }^{8}$, den er mehr als Mobilisierungsdiskurs sieht. Allein eine europäische Öffentlichkeit, neue Aktionen für die Jugend und eine neue Moralisierung des politischen Lebens helfen, dem Anwachsen von populistischen Bewegungen entgegenzuwirken. Der Philosoph Joël Gaubert geht noch einen Schritt weiter, beschreibt Populismus als Malaise in der gegenwärtigen Demokratie, die die tradierten Modelle der Repräsentation, Partizipation und Deliberation herausfordert.

In dasselbe Horn stößt die Sonderausgabe der Critique zu „den Populismen“. Hier geht es darum, den populistischen Appell an das als homogen konstruierte „Volk“ geisteswissenschaftlich, also auch literatur- und sprachwissenschaftlich einzubetten. Der „ismus“ des Wortes, wörtlich die dauerhafte Beschäftigung mit dem „Volk“, gibt ebenso Anlass zur Diskussion wie seine „pejorative“ Verwendung, wie Marie-Anne Paveau verdeutlicht. Dennoch ist der Tenor des Heftes, dass der Populismus mehr ist als eine bloße Worthülse, die man dem Gegner überstülpt, vielmehr als eine globale Erscheinung anzusehen ist. Das macht der bereits erwähnte Guy Hermet zumindest für Lateinamerika und Europa deutlich, indem er Konstanten und Mutationen des Populismus beleuchtet. Auch eine dritte akademische Fachzeitschrift, „Politique“, macht den Populismus zum Schwerpunktthema. Jérôme Jamin ortet ihn als polemisch angelegten Simplifizierungsdiskurs, der eine gewisse Rechtfertigung dadurch erhält, dass er wahre Probleme anspricht. Zwischen Populismus und NichtPopulismus bestehe oftmals ein schmaler Grat, wie das Beispiel von Nicolas Sarkozy zeige.

In der französischen Literatur überwiegt eine assoziative Herangehensweise, die weniger auf konkrete empirische Auswertungen zurückgreift und unter dem Einfluss der Präsidentschafts- und Parlamentswahlen 2012 steht. Populismus wird eher als Politikstil oder -strategie gesehen, nicht als Ideologie. In Frankreich hat der Populismus in der Politik einen festen 
Platz, auch historisch durch einen plebiszitären Cäsarismus von Napoleon III., der 1851 durch einen mittels eines Referendums ratifizierten „Staatsstreich“ an die Macht gekommen war, und durch den Poujadismus in den 1950er Jahren, der mit dem Front National fortgeführt wurde. Es lässt sich damit eine Genese des Populismus nachvollziehen.?

In den Niederlanden gab es hingegen lange keinen Populismus. Die Harmonie im Poldermodell fand sein Ende mit Pim Fortuyn, der bei den Parlamentswahlen im Jahre 2002 mit seiner Liste aus dem Stand 17 Prozent der Stimmen erzielte. Fortuyns Ermordung bremste den Siegeszug des Rechtspopulismus nur kurzzeitig, dessen Banner anschließend von der Freiheitspartei unter Geert Wilders weiter getragen wurde. Eben dieser Wilders, alleiniges Mitglied seiner Partei, sorgte dafür, dass vorgezogene Neuwahlen stattfinden mussten, da die Minderheitsregierung von seiner Tolerierung abhängig war.

Paul Lucardie und Gerrit Voerman aus Groningen machen klar, wie es dazu gekommen ist. Dabei fahnden sie nach populistischen Momenten in der Vergangenheit, die vielleicht helfen, die heutigen Phänomene zu verstehen. Ähnlich wie in Frankreich oder auch in Deutschland gibt es mit der Sozialistischen Partei eine Kraft, die deutliche linkspopulistische Züge aufweist, wie die Autoren in einem eigenen Kapitel verdeutlichen. Geert Wilders hat sich im Laufe der Zeit mehr und mehr radikalisiert. Er, proisraelisch und amerikafreundlich ausgerichtet, passt in mancherlei Hinsicht nicht in das klassische Bild. Er zeigt eine Islamophobie, die von apokalyptischen Szenarien und Verschwörungstheorien im Zusammenhang mit der Euroarabien-Ideologie geprägt ist, die eigentlich für Extremisten typisch sind. ${ }^{10}$ Kenntnisreich analysiert das Autorenduo alle Facetten des Erfolgs, den sie am Ende in den europäischen Zusammenhang setzen. Populistische Akteure werden im globalen Kontext, quer über die Kontinente hinweg identifiziert. Mehr und mehr kommt es dabei in einer vergleichenden Forschungsagenda zu einem Vergleich zwischen dem Populismus in Lateinamerika und den USA. ${ }^{11}$ Vermehrt wird dabei nicht nur vom Schaden, sondern mit differenzierter Betrachtung auch vom Nutzen des Populismus für die Demokratie geredet, zum Beispiel als ein mitunter notwendiger Stachel im Fleisch des politischen Establishments. Immer spielt dabei die Konzeptualisierung von Populismus eine Rolle, die allerdings von einem common sense weit entfernt ist. Das gilt auch für die Frage, was als populistisch beziehungsweise nicht-populistisch bezeichnet wird. Die Beispiele aus Frankreich und den Niederlanden weisen darauf hin, dass es in Zukunft weiterhin empirischen Bedarf gibt, das Populismuskonzept grundsätzlich anzuwenden und zu operationalisieren.

Florian Hartleb

9 Vgl. dazu Pierre Birnbaum, Genèse du Populisme. Le Peuple et les Gros, Paris 2010.

10 Diese auf Bat Yéors fußende Theorie besagt, dass europäische und arabische Politiker nach der Ölkrise des Jahres 1973 in einem europäisch-arabischen Dialog vereinbarten, dass arabische Immigranten im Tausch gegen Öl in Scharen nach Europa kommen und ihren Glauben verbreiten dürfen.

11 So auch der Tenor eines neuen Sammelbandes von Cas Mudde / Cristóbal Rovira Kaltwasser (Hrsg.), Populism in Europe and the Americas: Threat or Corrective for Democracy?, Cambridge / New York 2012. 